\title{
Practical issues related to uterine pathology: in situ and invasive cervical glandular lesions and their benign mimics: emphasis on cytology-histology correlation and interpretive pitfalls
}

\author{
David C Wilbur
}

James Homer Wright Laboratory of Pathology, Department of Pathology, Massachusetts General Hospital, Harvard Medical School, Boston, MA, USA

\begin{abstract}
In situ and invasive neoplastic glandular lesions of the uterine have cytologic correlates that must be distinguished from a variety of benign and reactive conditions. Careful study of the cytologic features allows discrimination in the majority of cases; however, the designation of 'atypical glandular cells' is reserved for equivocal cases that cannot be readily resolved. In this article, the cytologic features of endocervical adenocarcinoma in situ and invasive endocervical adenocarcinoma will be presented, highlighting their correlation to the well-known histologic features. Variants of the usual type of endocervical neoplasms that have important clinical and differential diagnostic features, including mucinous adenocarcinoma and clear-cell carcinoma, will be discussed. There are a number of common cytologic mimics of endocervical neoplasms, including tubal metaplasia, directly sampled (abraded) endometrium, and high-grade squamous intraepithelial lesion involving endocervical glands. The cytologic features of these entities and their differentiation from endocervical neoplasia will be explored. Finally, the role of ancillary studies such as human papillomavirus testing in the management of glandular lesions of the cervix will be integrated into the discussion.

Modern Pathology (2016) 29, S1-S11; doi:10.1038/modpathol.2015.138
\end{abstract}

Over the past quarter century, there has been an increasing recognition of glandular lesions of the cervix. Although well described in both invasive and in situ forms, in clinical practice these types of cancers were considered to be relatively rare, particularly when compared with their far more prevalent squamous counterparts. In the period 1976-1980, adenocarcinoma accounted for only $12 \%$ of all invasive cervical cancers. ${ }^{1}$ In situ carcinoma, while identifiable in histologic material, was rarely detected in cytologic specimens. With the sharp decline in cancers of squamous type, which followed widespread implementation of cervical cytology screening, and because of a perceived increase in

Correspondence: Dr DC Wilbur, MD, James Homer Wright Laboratory of Pathology, Department of Pathology, Massachusetts General Hospital, Harvard Medical School, 55 Fruit Street, Warren 120, Boston, MA 02114, USA.

E-mail: dwilbur@mgh.harvard.edu

Received 2 September 2015; revised 16 September 2015; accepted 5 November 2015 incidence of glandular cancers, possibly secondary to more prevalent hormone use, greater attention has been paid to their histo- and cytomorphologic criteria and to the specific subtypes of glandular cancer that can be identified. In addition, with the advent of improved Papanicolaou sampling devices, which gather greater amounts of cytologic material from the upper regions of the endocervical canal, the cytologic features of adenocarcinoma in situ (AIS), and of the less prevalent variants of invasive adenocarcinoma, have been better documented.

This review will discuss the cytologic features of the most common 'usual' type of endocervical adenocarcinoma, in conjunction with its correlative histomorphologic features. There are a number of common morphologic mimics of AIS and invasive adenocarcinoma, which can lead to overinterpretation in cytology specimens. These entities and their differential diagnostic features when compared with true cervical glandular neoplasia will be emphasized. A number of clinically important, but relatively rare, variants of invasive adenocarcinoma, 
including villoglandular, clear-cell and mucinous types, have important clinical management ramifications when compared with the usual type. These variants can and should be recognized in cytology specimens, and their clinical and cytologic features will be discussed. Finally, laboratory testing for human papillomavirus (HPV) is now commonly advocated for the detection of squamous lesions. As the majority of cervical glandular lesions are also associated with HPV, testing for the virus may also serve a purpose in their management regimens. The use of HPV testing, however, differs significantly for glandular neoplasia as compared with their squamous counterparts, and hence it is important to illustrate the differences and the rationale for its use.

\section{Endocervical neoplasia: general features}

Endocervical adenocarcinoma has shown a small but definitive increase in incidence over the past 40 years. ${ }^{1}$ Reagan and $\mathrm{Ng}^{2}$ found in the early 1940s that endocervical adenocarcinoma comprised $5 \%$ of all cervical carcinomas. In recent years, this percentage has increased to $20-25 \% .^{3}$ Most recent studies have found incidence rates to be between 14 and $34 \%$ of all cervical carcinomas. ${ }^{4}$ US SEER data gathered up to 2008 (Table 1) has shown that there has been a slight increase in the absolute rate of endocervical invasive carcinoma, but this rise has been overshadowed by the decreased absolute incidence of squamous carcinomas. ${ }^{5}$ In addition, international databases have shown steady rates or declines in endocervical adenocarcinoma, raising questions about the true trajectory of endocervical neoplasia prevalence.

Bousfield et $a{ }^{6}{ }^{6}$ in a large series comprising the spectrum of endocervical neoplasia, found that age and lesion severity were directly proportional, similar to that which has been noted for squamous neoplasia (Table 2). In addition, they found a significant predilection for cervical adenocarcinomas in young women, noting that $78 \%$ of cervical carcinomas arising in the first three decades of life were of endocervical origin.

The epidemiology of endocervical neoplasia differs from that of squamous neoplasia of the cervix.
As compared with squamous lesions, endocervical neoplasia arises more commonly in women of higher socioeconomic status, with higher education levels, lower unemployment, higher incomes, and show less linkage to early sexual intercourse and smoking. ${ }^{7}$ Similar to their squamous counterparts, the linkage of endocervical glandular neoplasia to infection by high-risk HPV is also strong. Up to $100 \%$ of AIS and $94 \%$ of invasive lesions have been shown to be positive for HPV when examined by sensitive detection techniques. ${ }^{8}$ A unique feature of endocervical neoplasia is a greater prevalence of HPV type 18. In all, 50-75\% of endocervical tumors are HPV type 18 positive, with the remainder being predominantly types 16 and the 18-associated type $45 .^{9}$ In particular, HPV 16 variants (ie, Asian-American) have been shown to be associated with endocervical neoplasia. It has been postulated (but not proven) that use of hormones increases the risk of endocervical neoplasia, similar to what has been proven for endometrial carcinomas. The association noted between the Asian-American variant of HPV type 16, which is known to have increased susceptibility to estrogenic effects, and increased incidence in obese individuals provides additional support for this linkage. ${ }^{10}$

A further link between endocervical and squamous carcinogenesis exists. About $50 \%$ of endocervical tumors have a coexisting squamous lesion present. ${ }^{11}$ Such data might suggest that both may arise from a common progenitor cell in the cervix, with differentiation along squamous, endocervical, or both lines based on such factors as HPV type, hormone use, and as-yet-undetermined cofactors.

Table 2 Endocervical neoplastic categories and age ${ }^{6}$

\begin{tabular}{lcc}
\hline & Age (years) & Age range \\
\hline EC ‘dysplasia’ & 36 & $33-38$ \\
AIS & 37 & $22-70$ \\
Microinvasive CA & 41 & $28-66$ \\
Invasive CA & 49 & $26-85$ \\
\hline
\end{tabular}

Abbreviations: AIS, adenocarcinoma in situ; CA, cancer; EC, endocervical.

Table 1 Cervical cancer incidence over 35 years in the United States ${ }^{\mathrm{a}}$

\begin{tabular}{|c|c|c|c|c|c|c|}
\hline & \multicolumn{2}{|c|}{ 1973-1989 } & \multicolumn{2}{|c|}{ 1990-2008 } & \multicolumn{2}{|c|}{ Change per 100000 women } \\
\hline & $\mathrm{N}(\%)$ & $95 \% C I$ & $\mathrm{~N}(\%)$ & $95 \% C I$ & EAPC & $95 \% C I$ \\
\hline Total & $19907(100.0)$ & - & $20456(100.0)$ & - & -2.3 & -2.5 to -2.1 \\
\hline SCC & $17113(86.0)$ & $85.5-86.4$ & 15504 (75.8) & $75.2-76.4$ & -3.0 & -3.2 to -2.1 \\
\hline ACA & 2797 (14.0) & $13.6-14.5$ & $4952(24.2)$ & $23.6-24.8$ & 0.6 & 0.2 to 1.0 \\
\hline
\end{tabular}

Abbreviations: ACA, adenocarcinoma; CI, confidence interval; EAPC, estimated annual percent change; $N$, number of cases; SCC, squamous cell carcinoma; SEER, surveillance, epidemiology, and end results.

${ }^{a}$ Data from the National Cancer Institute's SEER Program. ${ }^{5}$ 
Clinically, preinvasive neoplasms of endocervical origin are generally asymptomatic. They may be visible on colposcopy if they involve the transformation zone, but higher in the canal they are often non-visible. Even in invasive endocervical carcinomas, $20-30 \%$ of colposcopies may be interpreted as normal. Symptoms of abnormal uterine bleeding are most common; occasionally women have vaginal discharge or pain. In asymptomatic cases, the tumor is generally discovered via a routine cervical cytology specimen; however, the sensitivity of detection is low in comparison with squamous lesions, as would be expected by their location generally higher in the endocervical canal, above the transformation zone. Australian cancer registry data showed that upon retrospective review of cytology following a histologic diagnosis of AIS, the false-negative rate was about $50 \% .{ }^{12}$ Causes for false-negative examinations were predominantly sampling (meaning no identifiable lesion cells were found on the slide), and also interpretation (meaning that upon review, lesional cells were present that had been missed or misinterpreted as benign or reactive). For invasive endocervical adenocarcinoma, the false-negative rate of preceding cytologic examination was found to range from 45 to $74 \%$, with about $20 \%$ being due to interpretation errors. ${ }^{13}$ Suffice it to say that cytology alone is significantly less sensitive for glandular, as opposed to squamous lesions, and that education and experience is required to achieve best detection sensitivity. The cytologic criteria for the diagnosis of AIS were not well documented even as late as the first edition of the Bethesda System in $1988,{ }^{14}$ in which it was subsumed under the category of 'glandular atypia'. At the time of the second edition in 2001, ${ }^{15}$ AIS was specifically incorporated as a diagnostic entity because in the ensuing years, the morphologic criteria had been well studied and documented, and they had been found to be reasonably well reproduced between observers. At that time, it was hoped that improvements in detection sensitivity from the use of new sampling devices and enhanced recognition and proper categorization would ensue. Fortunately, this appears to have occurred as recent data suggests that up to $80 \%$ of women having either AIS or invasive endocervical adenocarcinoma will have an abnormal cervical cytology specimen ranging from at least 'atypical' (for an incomplete, but compelling morphologic pattern) to a more specific interpretation. ${ }^{13}$ In addition, the prevalence of AIS in registry data is increasing at a greater rate than are the precursor squamous lesions (sevenfold increase in SEER data in the past 20 years). ${ }^{16}$ This increase is almost certainly due to a compilation of the factors noted above, including improved sampling devices, proper sampling technique, introduction of liquid-based cytology preparation allowing for improved sample preservation and presentation, and finally from better recognition of the cytologic features of glandular neoplasia.
The addition of HPV testing has the potential to substantially improve detection of endocervical neoplasia. As noted above, virtually all of the most common types of AIS and invasive endocervical adenocarcinoma are HPV associated. Primary cervical cancer screening via the use of HPV cotesting (Pap test plus HPV testing) or, more recently, by HPV testing alone have shown more sensitive detection of glandular lesions. ${ }^{17} \mathrm{HPV}$ primary screening, however, will not detect the rare forms of adenocarcinoma that are not associated with HPV (eg, mucinous carcinoma) as will be described below. For this reason, some have advocated the use of cotesting, or at least cytology at some (as-yet-unspecified) interval to achieve maximal screening benefit.

\section{Endocervical AIS}

AIS generally presents without symptoms. It may be inapparent on colposcopic examination as it most often involves the endocervical canal. It is most often unifocal and hence continuous, although rare examples of multifocal disease may be identified. ${ }^{18}$ It is therefore most often first detected via screening procedures, most commonly Papanicolaou testing or by the less specific process of HPV testing. HPV testing is more sensitive, but requires colposcopy and biopsy to identify the disease. On histopathology specimens, AIS presents as a hyperchromatic epithelium involving both superficial and deep glands. These hyperchromatic cells may be admixed, with benign endocervical epithelium showing a sharp transition (Figure 1). In contrast to the normal simple endocervical epithelium, AIS shows pseudostratification of enlarged hyperchromatic

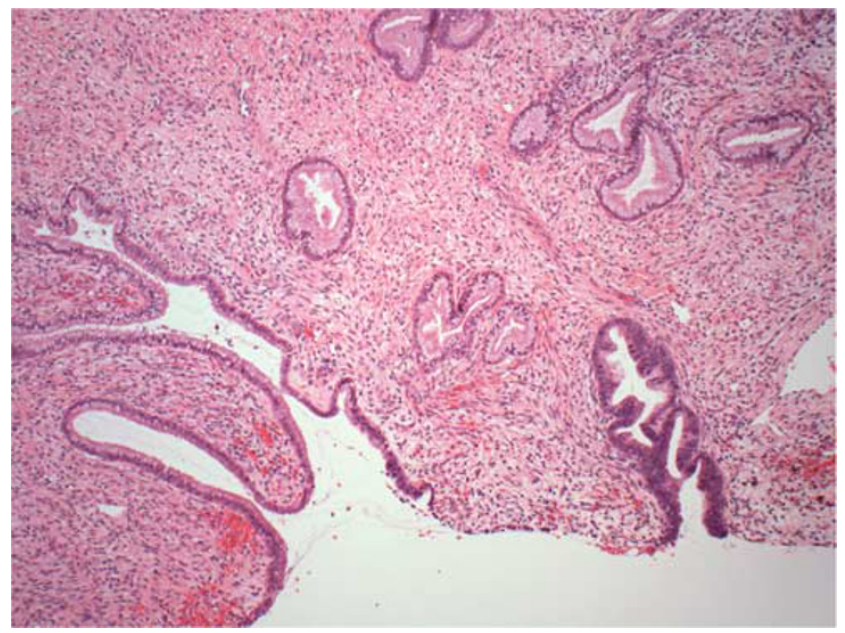

Figure 1 Endocervical adenocarcinoma in situ: low magnification of endocervical adenocarcinoma in situ involving a single gland. The gland in the lower right shows a hyperchromatic epithelium in stark contrast to the normal endocervical epithelium lining the non-involved glands and surface. This hyperchromatic area is often the first identifiable feature. 


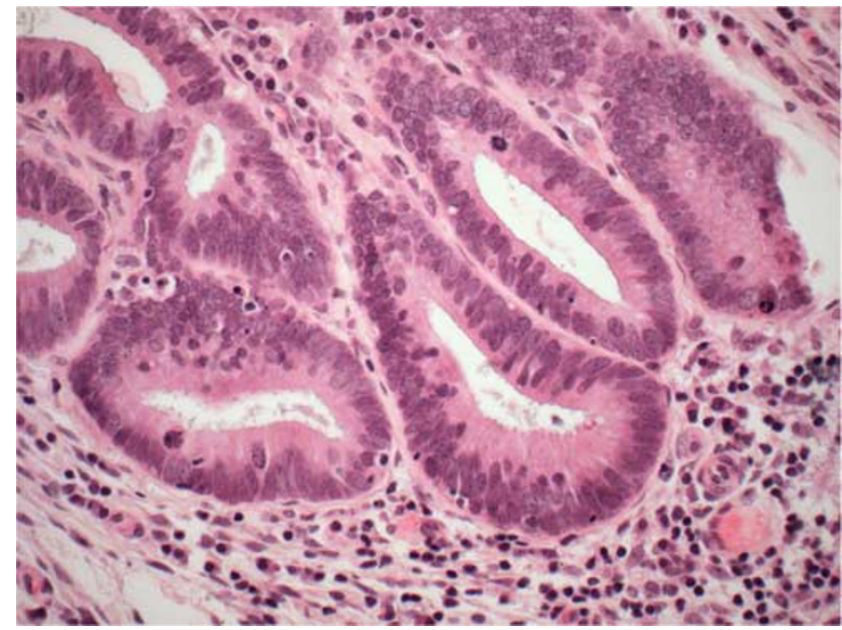

Figure 2 Endocervical adenocarcinoma in situ: note the pseudostratified nuclei with numerous mitotic figures present.

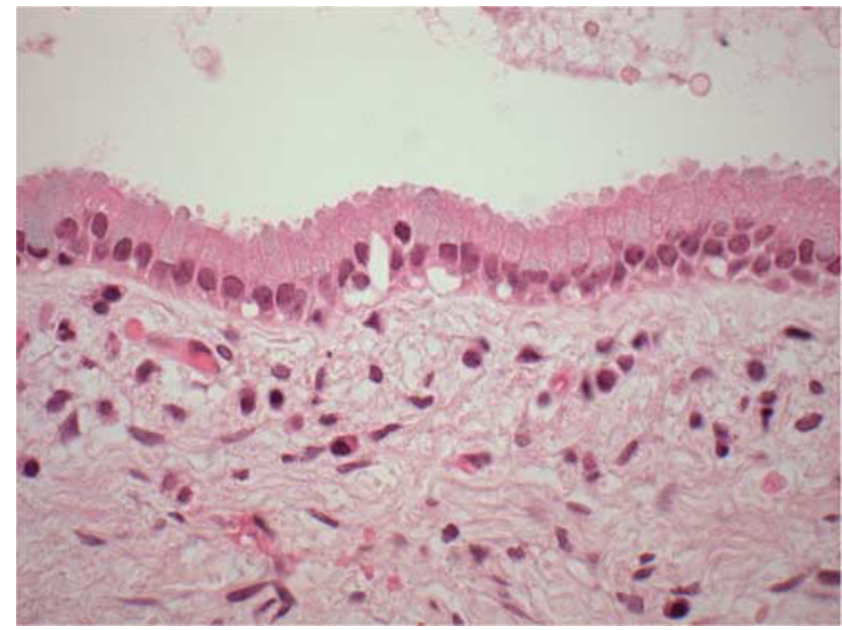

Figure 3 Normal endocervical epithelium: this epithelium consists of a single layer of columnar cells with basal nuclei and mucinous caps. Contrast to the pseudostratified epithelium of adenocarcinoma in situ (AIS) present in Figure 2.

nuclei, numerous mitotic figures (often apical), and apoptosis (Figure 2).

To understand the cytologic features of AIS, those of the normal endocervical epithelium must also be well appreciated. In the transformation zone, endocervical epithelium has a simple columnar configuration with relatively uniform nuclei present at the basal portion of each cell with an overlying cap of abundant frothy mucinous cytoplasm (Figure 3). This histologic appearance translates in a cytologic specimen to groups of two configurations: (1) the linear 'picket fence' arrangement with columnar cells lining up completely analogous to the histology as presented (Figure 4); and (2) the two-dimensional 'honeycombed' sheet, when abraded cells are observed 'end on' viewing down the long columnar

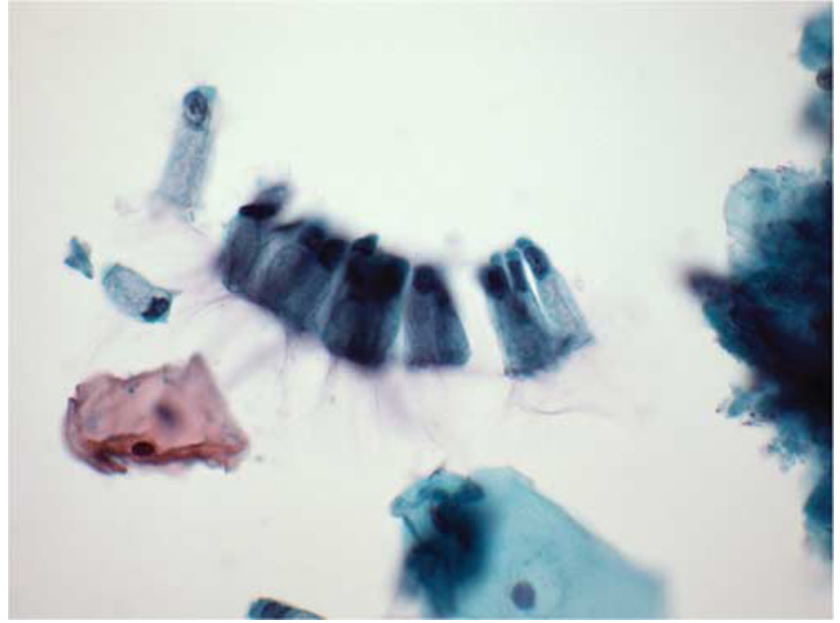

Figure 4 Normal endocervical cells: a 'picket fence' configuration recapitulating the histology of Figure 3.

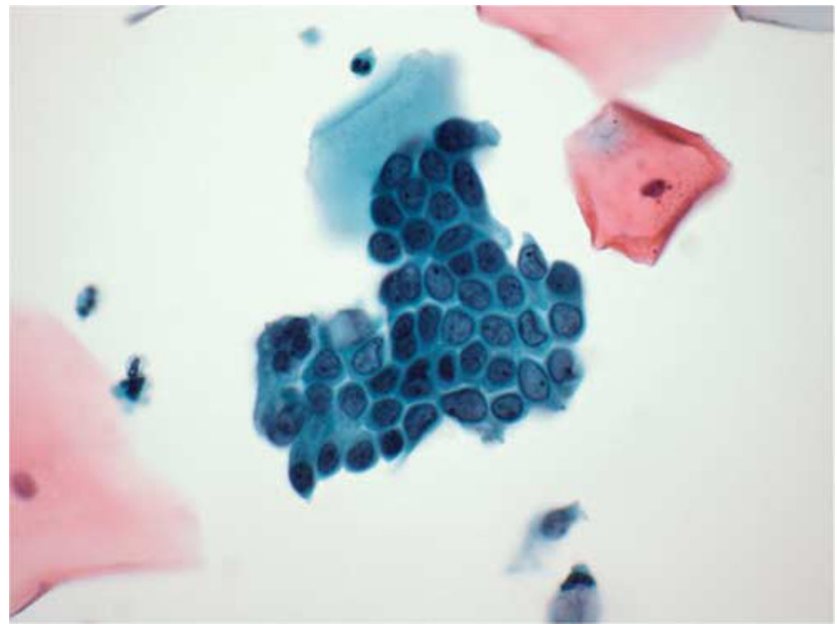

Figure 5 Normal endocervical cells: ‘honeycomb' architecture for normal endocervical cells when viewed end-on. Note the significant pleomorphism of the endocervical nuclei, which is a characteristic reactive change, not to be taken in and of itself as a feature of neoplasia.

axis (Figure 5). In the latter, nuclei are evenly dispersed with little or no overlap, and the mucous caps of the cells are identified in focal planes above (or below) the nuclei. The appearance of normal endocervical cells changes as upper portions of the endocervical canal are sampled. As the endocervical merges with the endometrial mucosa, the epithelium becomes pseudostratified with generally smaller nuclei (Figure 6). In addition, tubal metaplastic change becomes more common, which can in and of itself create differential diagnostic difficulties (discussed below).

The cytologic features of AIS translate directly from the histopathologic features noted above, and are compiled in Table 3. Cytologic specimens are cellular with increased numbers of dense 


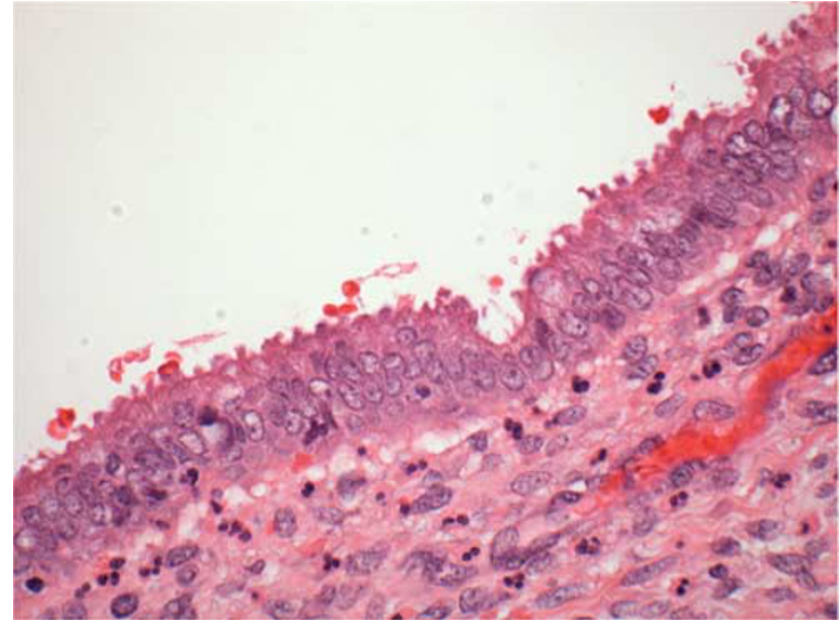

Figure 6 High canal endocervical epithelium: when endocervical epithelium begins to merge with lower uterine segment endometrial epithelium, it becomes increasingly pseudostratified and the mucous cap is depleted. Cells sampled from these areas are more likely to cause over interpretations of atypical glandular cells because of their morphologic similarities with endocervical neoplasia. hyperchromatic groups (the so-called 'hyperchromatic crowded groups' or 'HCGs'). Groups show prominent 'nuclear feathering' or protrusion at the margins (Figure 7). The chromatin of the enlarged nuclei is evenly distributed, but shows coarse granularity, a feature that is very helpful in distinguishing AIS from benign mimics such as tubal metaplasia (discussed below).

Variants of AIS are uncommon and can be challenging to identify when present. Endometrioid AIS is particularly difficult to detect because the nuclei are smaller than in usual AIS; hence, cell groups will more closely resemble direct sampling or abraded endometrial cells (Figure 8). The intestinal variant has some distinctive features when compared with the usual type. It occurs at an older age by an average of 10 years, it is less commonly HPV and p16 positive (suggesting that some may have an alternative (non-HPV) pathogenesis), can show less proliferation by Ki67 labeling, and is immunohistochemically more akin to intestinal neoplasia (CDX2-positive). ${ }^{19}$

Table 3 Cytologic features of AIS, endocervical adenocarcinoma, and their mimics

\begin{tabular}{|c|c|c|c|c|c|}
\hline & AIS & $\begin{array}{l}\text { Invasive endocervical } \\
\text { adenocarcinoma }\end{array}$ & $\begin{array}{l}\text { Tubal } \\
\text { metaplasia }\end{array}$ & $H S I L$ & Directly sampled endometrium \\
\hline Cellular groups & Yes & Yes & Yes & Yes & Yes \\
\hline Pseudostratified strips & Yes & Yes & Yes & No & Yes \\
\hline Feathering & Yes & Yes & No & No & No \\
\hline Rosettes & Yes & Yes & No & No & No \\
\hline Nuclear overlap & Yes & Yes & No & Yes & No \\
\hline Coarse chromatin & Yes & Yes & No & Yes & No but can be with degeneration \\
\hline Chromatin clearing & No & Yes & No & No & No \\
\hline Mitoses & Yes & Yes & Rare & Yes & Yes in proliferative phase \\
\hline Apoptosis & Yes & Yes & No & Yes & No \\
\hline Organoid groups with attached stroma & No & No & No & No & Yes \\
\hline Tumor diathesis & No & Yes & No & No & No \\
\hline
\end{tabular}

Abbreviations: AIS, adenocarcinoma in situ; HSIL, high-grade squamous intraepithelial lesion.
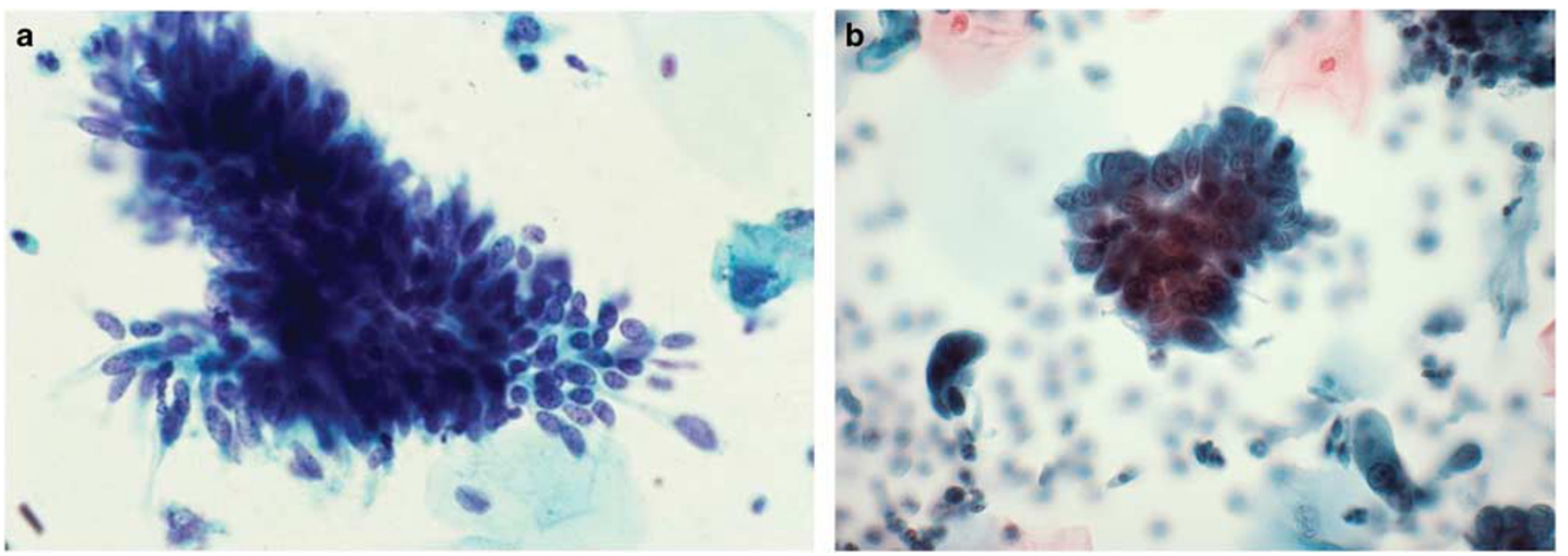

Figure 7 Endocervical adenocarcinoma in situ: (a) nuclear protrusion at the margins of the group-the so-called 'feathering' and (b) coarse granularity of the chromatin and the irregularity of the nuclear envelope. 


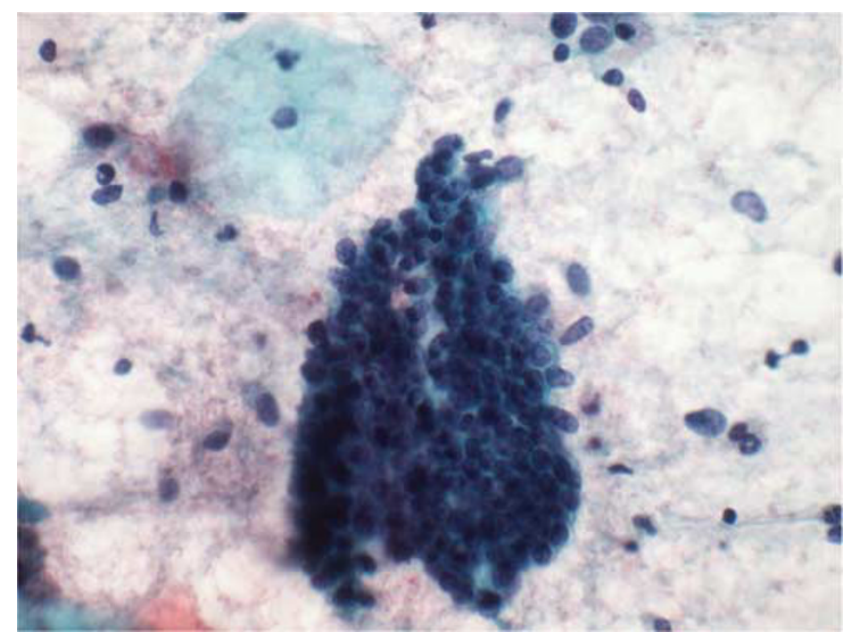

Figure 8 Endometrioid variant of endocervical adenocarcinoma: This entity can be difficult to identify because of the small size of the nuclei in contrast to the usual types. Feathering is still evident. These cells are often misinterpreted as being from benign endometrial sampling or exfoliation.

\section{Invasive endocervical adenocarcinoma}

In the 2014 WHO classification there are a number of types of invasive endocervical adenocarcinomas. ${ }^{20}$ The vast majority (up to $90 \%$ ) are of the usual type. Patients with endocervical adenocarcinoma generally present with abnormal uterine bleeding, but if small may be asymptomatic. On histologic examination, the usual type is most commonly of intermediate grade, composed of medium-sized glands lined by pseudostratified columnar cells. Cribriform glands may be present, and if appreciable in small superficial lesions may be a good indication of invasion. The cytoplasm is typically mucin-poor, and mitoses and apoptotic debris are commonly seen (Figure 9).

In cytologic preparations, the usual type of adenocarcinoma presents with abundant cellularity and, if well to moderately differentiated, many architectural features of AIS, including pseudostratification, rosettes, and feathered groups. In contrast to AIS, the chromatin is typically coarse and heterogeneous with areas of chromatin clearing. The background of the slide will show evidence of breakdown, inflammation, or necrosis-the so-called tumor diathesis (Figure 10).

A number of rare, but important variants of endocervical adenocarcinoma should be identified when possible. Villoglandular adenocarcinoma occurs in a younger population when compared with the usual type (mean 35 years), is typically well differentiated, and shows exophytic growth with minimal stromal invasion (Figure 11). It therefore has a more favorable outcome than the usual endocervical type, and in this younger population more likely to desire fertility, can be managed conservatively via cone excision only, if all of the

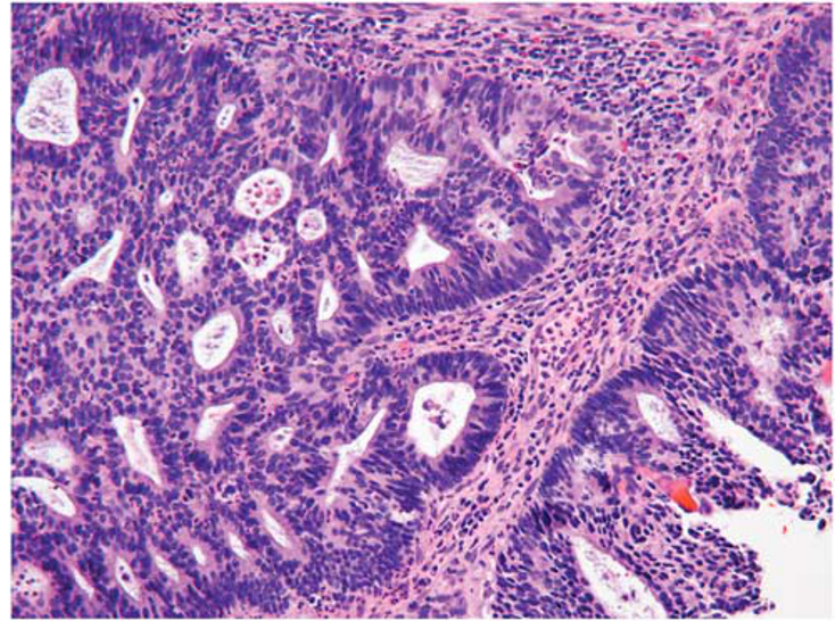

Figure 9 Endocervical adenocarcinoma, usual type: some features of adenocarcinoma in situ (AIS) remain with more severe cytologic change, architectural gland formations, and stromal reaction.

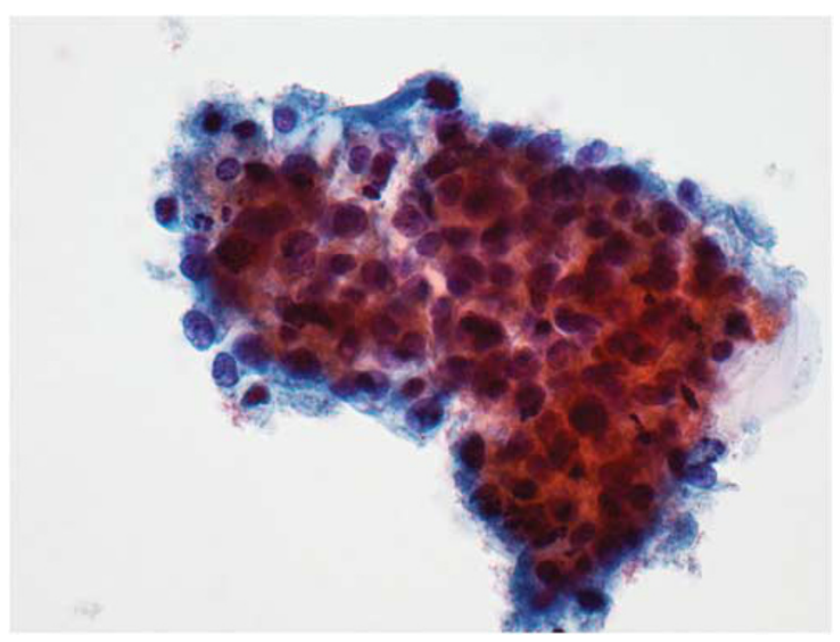

Figure 10 Endocervical adenocarcinoma, usual type: in contrast to Figure 7, nuclear atypia is greater than in adenocarcinoma in situ (AIS) with a prominent tumor diathesis (necrotic material and blood).

above criteria are met, no conventional adenocarcinoma is present, and if the margins are free of disease, and no lymphovascular invasion is identified. Cytologically, villoglandular adenocarcinoma presents as dense three-dimensional groups, often with bulbous projections ${ }^{21}$ (Figure 12). Mucinous adenocarcinoma is also uncommon but has more frequently been reported in the Japanese population (up to $25 \%$ of endocervical adenocarcinoma). The gastric type is most common, but intestinal and signet-ring types have also been reported. Mucinous adenocarcinoma is typically NOT HPV-associated. Its neoplastic pathogenesis is via the gastric route, hence it makes pyloric-type mucins (MUC6 and 


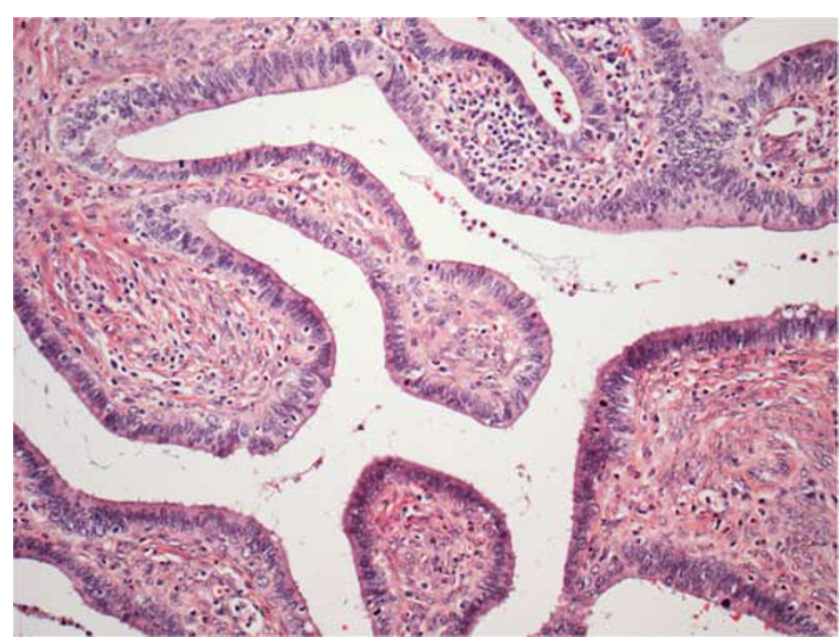

Figure 11 Villoglandular variant of endocervical adenocarcinoma: this designation is reserved for grade 1 lesions with single layers of abnormal epithelium covering stromal papillae.

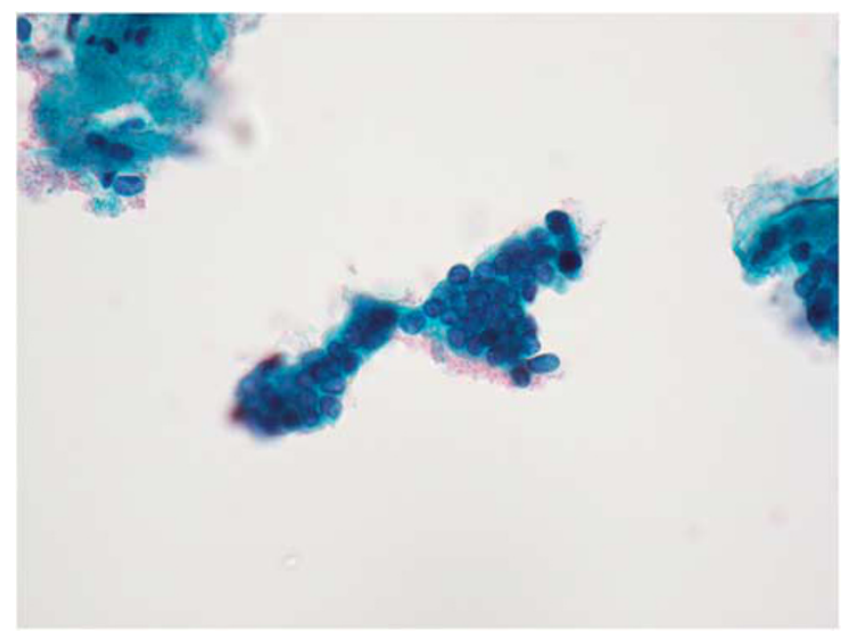

Figure 12 Villoglandular variant of endocervical adenocarcinoma: the cytology shows slender papillary groups consisting of 'grade 1' neoplastic cells.

HIK1083), and commonly shows STK11 mutations (chromosome 19p associated with Peutz-Jeghers syndrome). ${ }^{22}$ Mucinous carcinoma has a known noninvasive precursor lesion, lobular endocervical glandular hyperplasia (Figure 13). In histologic specimens, mucinous carcinoma commonly presents as tall mucin-producing glands penetrating the cervical wall with minimal if any stromal reaction and minimal cytologic atypia, hence the synonyms 'adenoma malignum' or 'minimal-deviation adenocarcinoma' (Figure 14). Cytologically, mucinous carcinomas are difficult to identify because of their morphologic similarity to benign mucinous endocervical epithelium. However, the

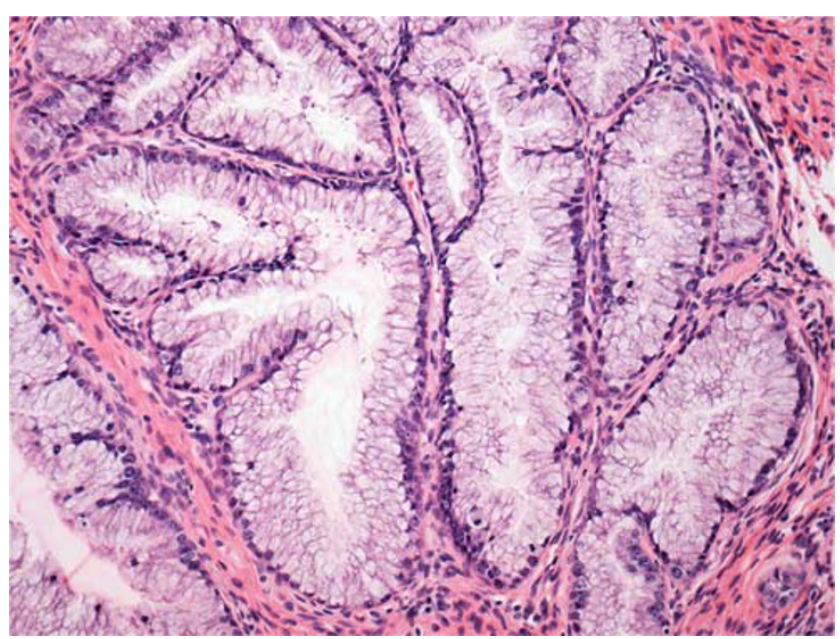

Figure 13 Lobular endocervical hyperplasia: this is a precursor lesion to mucinous (gastric-type) adenocarcinoma of the endocervix. Abundant complex mucinous epithelial cells are arranged in a lobular pattern without significant cytologic atypia are key features of this entity.

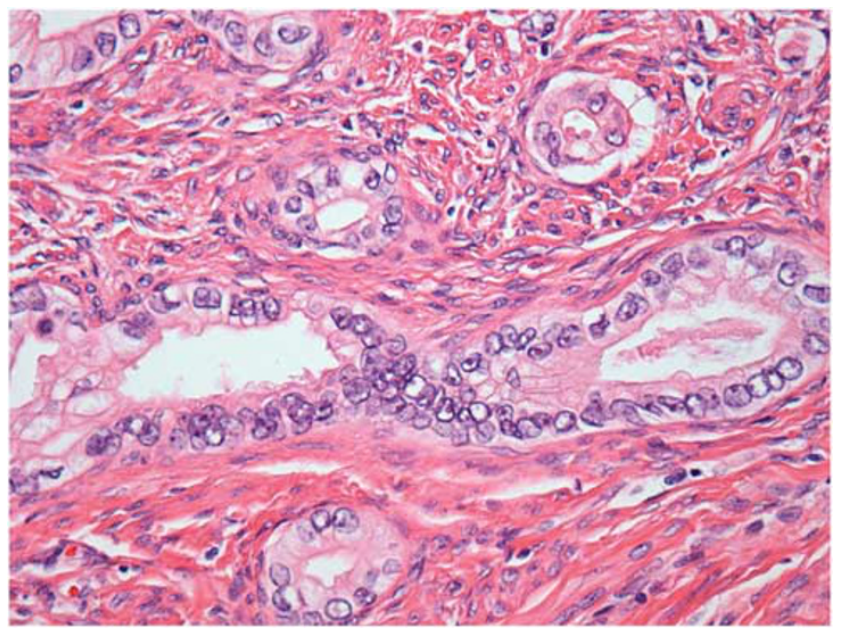

Figure 14 Mucinous (gastric type) endocervical adenocarcinoma: the malignant counterpart of lobular endocervical hyperplasia. Atypical mucinous epithelial cells are arranged in small- to medium-sized glandular units penetrating the cervical wall with little to no stromal reaction.

presence of large numbers of endocervical mucinous-type cells should prompt a closer examination for cytologic atypia (nuclear enlargement and irregularity), which is invariably present in some cells. In addition, the pyloric mucin will show a golden-brown appearance in the Papanicolaou stain and may hence be very informative if present (Figure 15). Primary clear-cell carcinoma in the endocervix is rare. Its histology is similar to other sites in the female genital tract. The far more common entity of endocervical microglandular hyperplasia can be a good mimic of clear-cell carcinoma. Both entities have closely packed glands, cystic glands, and infiltrating inflammatory cells. 
However, clinically they have very different presentations: clear-cell carcinoma in the older age group with symptoms and a mass, and microglandular hyperplasia in young reproductive age patients who are asymptomatic and have no mass, except occasionally a benign-appearing endocervical polyp. Clear-cell carcinoma shows high-grade nuclear atypia, whereas microglandular hyperplasia shows bland nuclei with characteristic subnuclear vacuoles (Figure 16).

Some investigators have suggested that neoplastic lesions less than AIS can be detected, and have suggested a designation of 'endocervical dysplasia' or 'low-grade endocervical neoplasia'. ${ }^{23}$ Although most agree that such 'dysplastic' lesions exist, reliable cytologic or histologic identification is not possible because of the wide range of non-neoplastic conditions that can present in the endocervical canal. Hence, the 2014 Bethesda System for cytologic interpretation prefers the use of the term 'atypical glandular cells' for cytologic presentations, which meet some but not all of the criteria for AIS. ${ }^{24}$ Some cases of atypical glandular cells will have follow-up showing true endocervical neoplasia, but a significant number will show either squamous neoplasia or benign reactive outcomes. The ability to distinguish between neoplastic and benign presentations can be difficult and hence further clinical evaluation is recommended for all such instances.

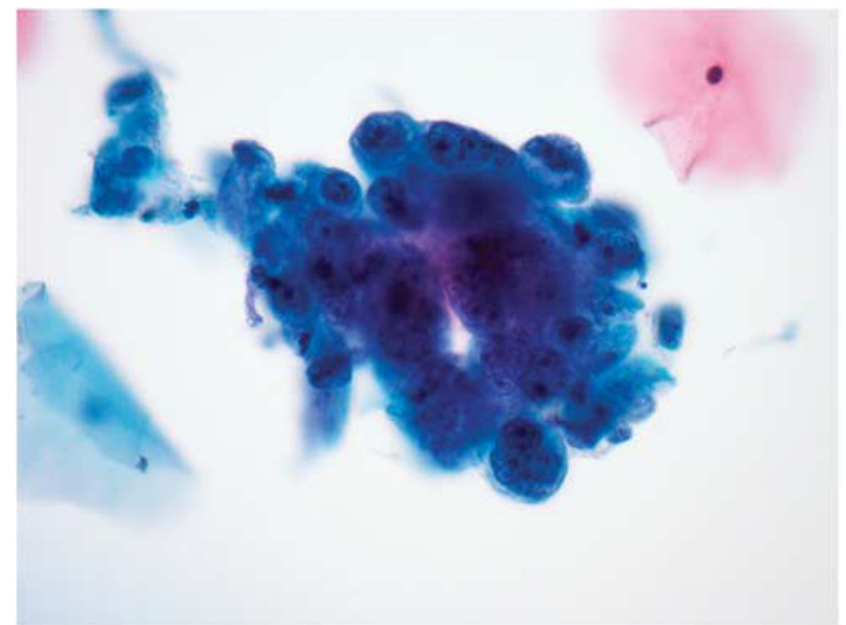

Figure 15 Mucinous (gastric type) endocervical adenocarcinoma: the cells contain abundant frothy mucinous epithelium and are arranged in groups or strips. Nuclear atypia is prominent.

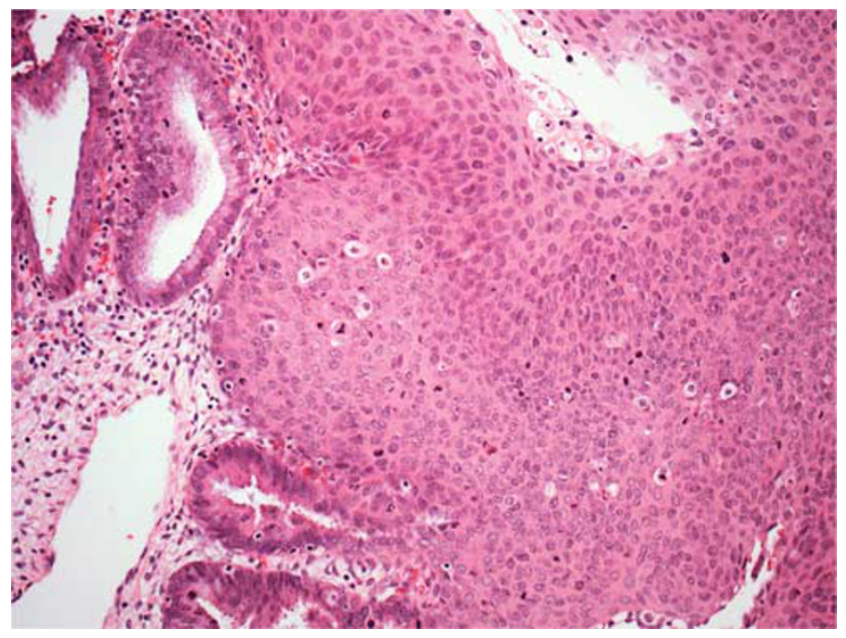

Figure 17 High-grade squamous intraepithelial lesion: when high-grade squamous intraepithelial lesion (HSIL) involves endocervical glands, its appearance in cytologic preparations can often show similarities to glandular lesions (see Figure 18).
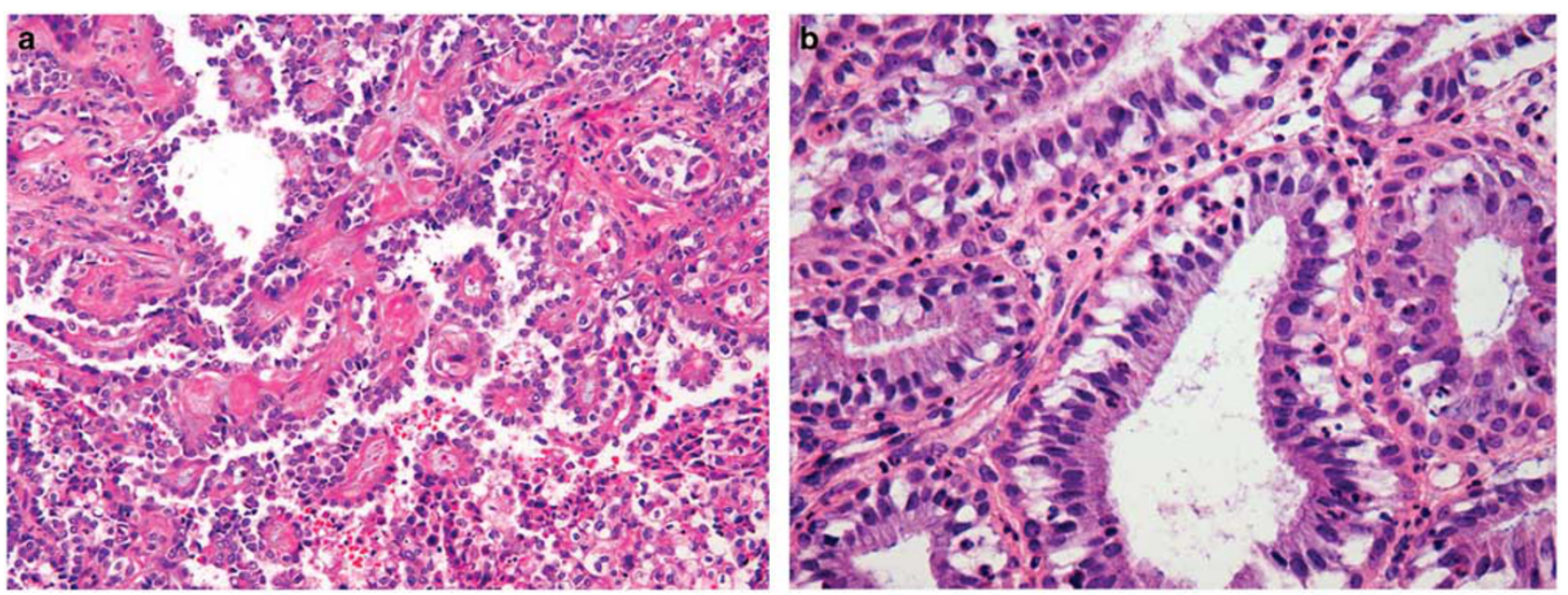

Figure 16 (a) Clear-cell carcinoma: marked cytologic atypia in densely packed glands with cleared or eosinophilic cytoplasm. (b) Microglandular hyperplasia: densely packed glands can show papillary structures with little nuclear atypia. The diffuse basal vacuoles are prominent. 


\section{Cytologic mimics of endocervical neoplasia}

There are a number of important mimics of endocervical neoplasia found in cervical cytologic specimens. These include high-grade squamous intraepithelial lesions (HSILs), tubal metaplasia, and cervical endometriosis/directly sampled endometrium (Table 3).

When HSIL involves the endocervical gland necks (Figure 17), the cells sampled may show some features of endocervical neoplasia, including densely packed hyperchromatic groups, palisading of cells, and pseudocolumnar configurations. Common features also include mitoses, apoptotic debris, and nuclear atypia. Important differential diagnostic features include lack of typical architectural of AIS described above, meaning no rosettes, feathering, or pseudostratified strips. In addition, if HSIL in a gland is suspected, review of the background for classic isolated HSIL cells is warranted-their presence makes HSIL far more likely as a cause for the dense groups identified (Figure 18).

Tubal metaplasia is a benign metaplastic process very common to both the endometrial and upper endocervical epithelium. Classic studies have shown that the newer sampling devices used in cervical cytology (broom and brush devices) sample many cells from the upper endocervical canal. ${ }^{25}$ Tubal metaplasia recapitulates the epithelium of the normal fallopian tube and is comprised of ciliated columnar cells, mucinous peg cells, and slender intercalated cells. This combination can lead to dense hyperchromatic groups, with pseudostratified architecture (Figure 19). Mitoses can be present, but apoptotic debris is not typically seen. Cilia are an important clue to its benign nature. However, tubal

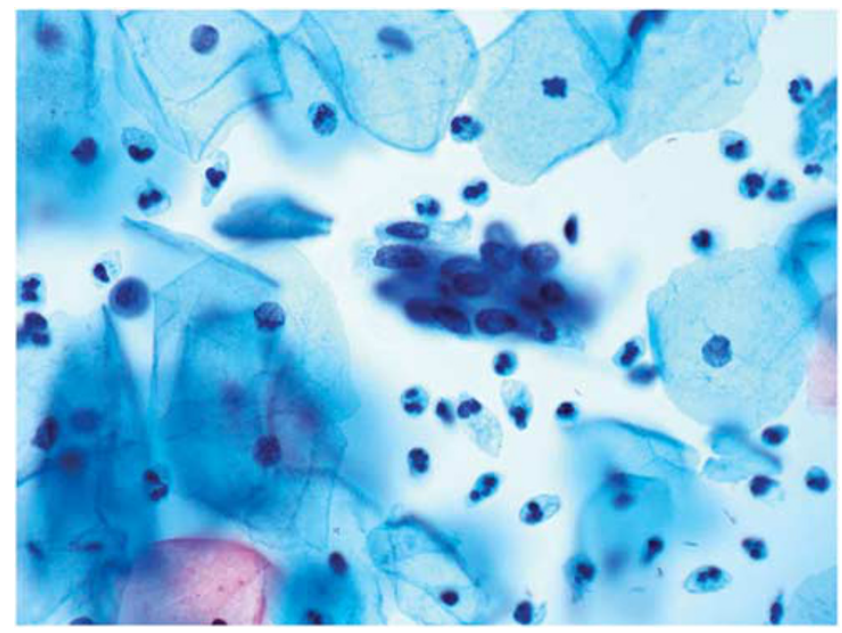

Figure 18 High-grade squamous intraepithelial lesion: hyperchromatic crowded groups with palisading and columnar configurations and prominent nucleoli are noted when high-grade squamous intraepithelial lesion (HSIL) involves the endocervical glands. metaplasia can coexist with AIS, hence the presence of cilia in some cells does not preclude that other atypical cells in the specimen may be neoplastic. Key to the differential between tubal metaplasia and AIS is examination of the chromatin pattern. Fine granularity favors tubal metaplasia, whereas coarse granularity favors AIS (Figure 20).

Direct sampling of endometrial tissue, be it from cervical endometriosis or lower portions of the uterine corpus, can create difficulty in cytologic differential diagnosis. Abraded endometrium can present in densely packed glandular groups, which can show pseudostratification, marked hyperchromasia, and coarse chromatin granularity. If the endometrial tissue is cycling or disordered, it can be mitotically active and rarely show apoptosis (Figure 21). The presence of large organoid groups of cells forming tubules, isolated groups of endometrial stromal cells, or stromal cells attached to the edges of the organoid groups is key to this differential diagnosis. Stromal cells attached to organoid groups can mimic feathering, but close examination will show the mesenchymal nature of these cells with wispy cytoplasm and small nuclear size. Overall, nuclei of directly sampled endometrium will be much smaller than those of AIS and attention to this particular detail should allow their distinction (Figure 22).

\section{Conclusions}

Endocervical neoplasia is increasing relatively, and also in absolute incidence, when compared with squamous neoplasia. Endocervical lesions are less likely to show symptoms or be colposcopically evident. Hence, cytology and primary HPV testing have a very important role in their detection. At present, all glandular atypias and definitive

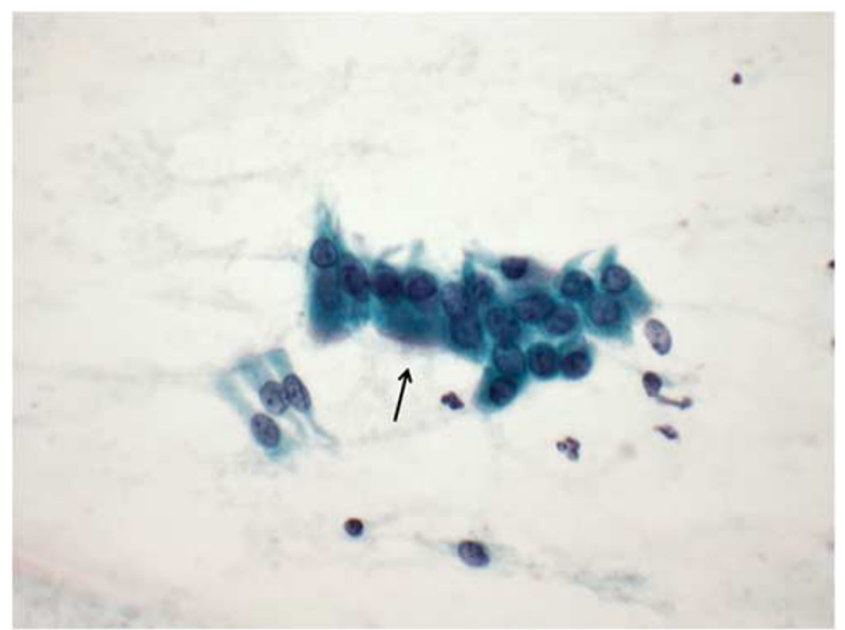

Figure 19 Tubal metaplasia: note the luminal border with a terminal bar and cilia (arrow). The smooth chromatin in this example is characteristic and should be compared with the coarse chromatin of adenocarcinoma in situ (AIS) (see Figure 7b). 

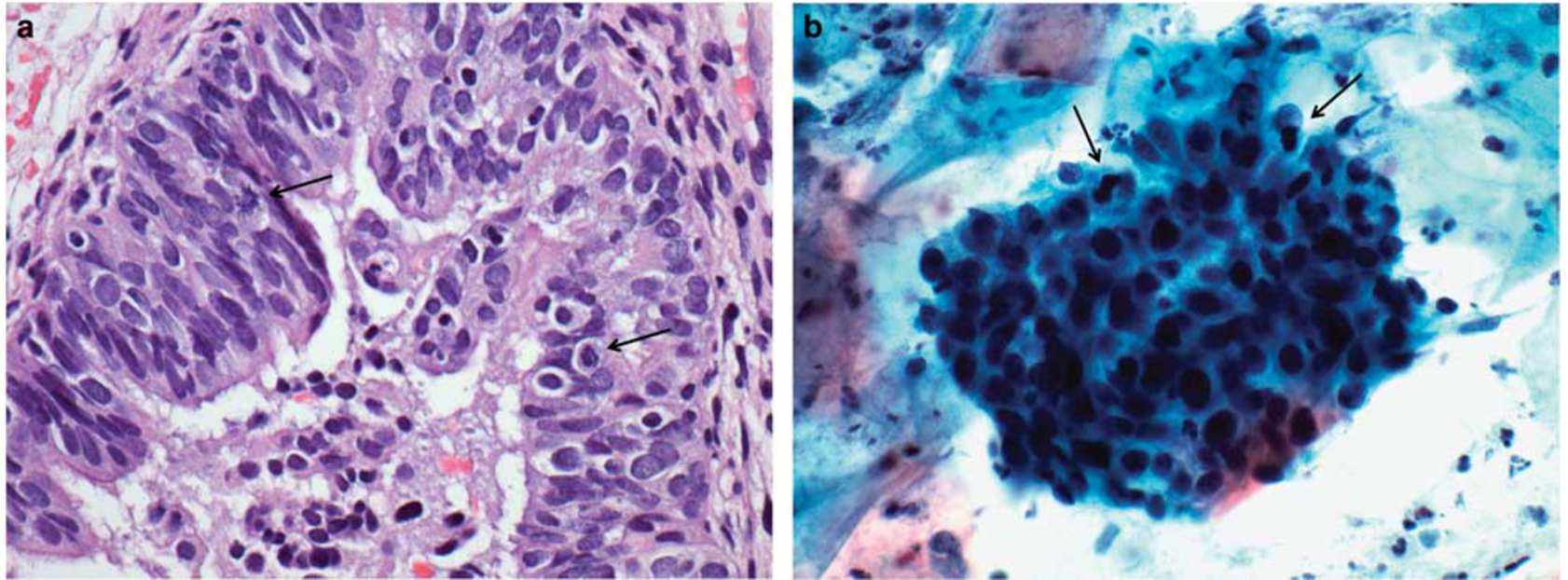

Figure 20 (a) Tubal metaplasia: there are several mitotic figures present (arrows). (b) Tubal metaplasia: hyperchromatic crowded group with mitotic figures (arrows). As in Figure 19, the chromatin pattern of the nuclei is smooth and evenly distributed in contrast to adenocarcinoma in situ (AIS).
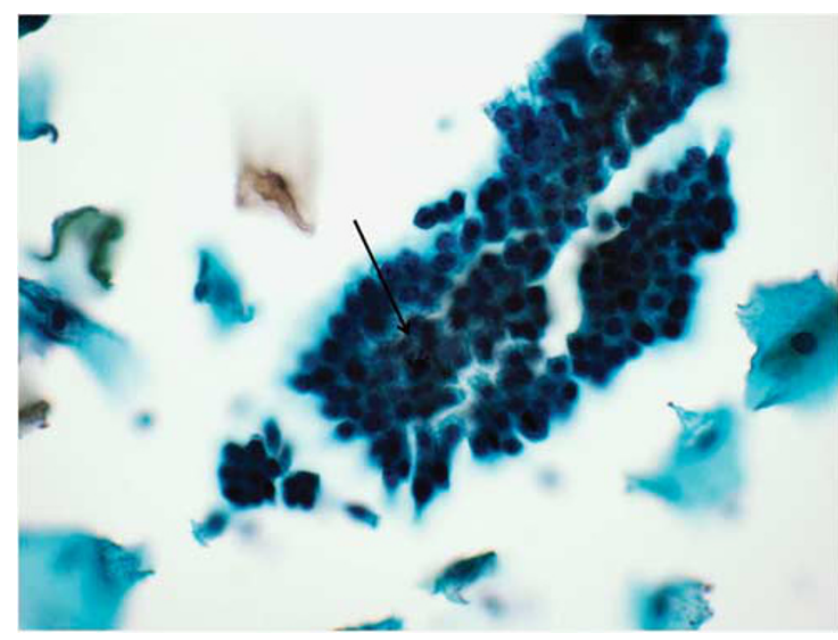

Figure 21 Directly sampled endometrium: mitotic figures are noted in the proliferative phase (arrow). Note the small size of the nuclei and the presence of endometrial stromal fragments.

neoplastic growths require colposcopic examination. ${ }^{26}$ Triage to colposcopy by HPV testing is not recommended owing to the fact that some endocervical carcinomas are HPV-negative, such as the mucinous variant.

A number of important cytologic mimics of endocervical neoplasia exist, which can lead to false-positive interpretations. These include tubal metaplasia and direct sampling of endometrium, which are both quite common. Careful attention to differential diagnostic features typically leads to the correct diagnosis, but in some scenarios it is best to classify such cases as 'atypical' to prompt further investigation.

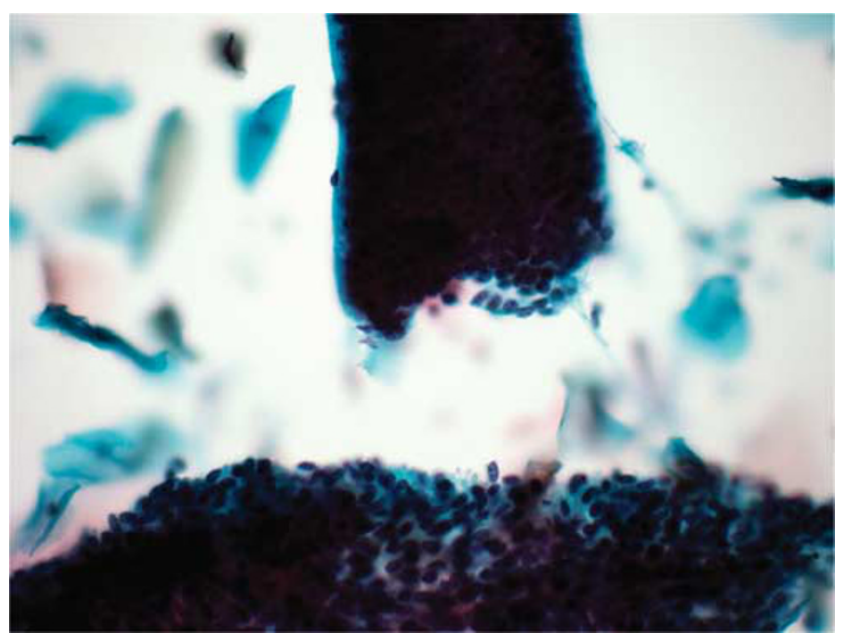

Figure 22 Directly sampled endometrium: a sharply defined hollow organoid tube of endometrial epithelial cells is present in the top of the field. A large fragment of spindled endometrial stromal cells is noted at the bottom of the field. The presence of both elements is useful in differentiating these cells from adenocarcinoma in situ (AIS).

\section{Disclosure/conflict of interest}

The author declares no conflict of interest.

\section{References}

1 Wang SS, Sherman ME, Hildesheim A et al. Cervical adenocarcinoma and squamous cell carcinoma incidence trends among White women and Black women in the United States for 1976-2000. Cancer 2004;100: 1035-1044.

2 Reagan JW, Ng ABP. Cellular detection of glandular neoplasms of the uterine cervix. In: Wied GL (eds). 
Compendium of Diagnostic Cytology, 6th (edn). Chicago, IL, USA: Tutorials of Clinical Cytology, 1988, pp 146-153.

3 Adegoke O, Kulasingam S, Virnig B. Cervical cancer trends in the United States: a 35-year population-based analysis. J Womens Health 2012;21:1031-1037.

4 Vizcaino AP, Moreno V, Bosch FX et al. International trends in the incidence of cervical cancer: I. Adenocarcinoma and adenosquamous cell carcinomas. Int J Cancer 1998;75:536-545.

5 Tambouret RT, Wilbur DC. Glandular Lesions of the Uterine Cervix. Cytopathology with Histologic Correlates. New York, NY, USA: Springer, 2015.

6 Bousfield L, Pacey F, Young Q et al. Expanded cytologic criteria for the diagnosis of adenocarcinoma in situ of the cervix and related lesions. Acta Cytol 1980;24:284-296.

7 Korhonen MO. Epidemiological differences between adenocarcinoma and squamous cell carcinoma of the uterine cervix. Gynecol Oncol 1980;10:312-317.

8 Zielinski GD, Snijders PJ, Rozendaal L et al. The presence of high-risk HPV combined with specific p53 and p16INK4a expression patterns points to high-risk HPV as the main causative agent for adenocarcinoma in situ and adenocarcinoma of the cervix. J Pathol 2003;201:535-543.

9 Clifford G, Franceschi S. Members of the human papillomavirus type 18 family (alpha-7 species) share a common association with adenocarcinoma of the cervix. Int J Cancer 2008;122:1684-1685.

10 Tornesello ML, Losito S, Benincasa G et al. Human papillomavirus (HPV) genotypes and HPV16 variants and risk of adenocarcinoma and squamous cell carcinoma of the cervix. Gynecol Oncol 2011;121: $32-42$.

11 Ayer B, Pacey F, Greenberg M et al. The cytologic diagnosis of adenocarcinoma in situ of the cervix uteri and related lesions. I. Adenocarcinoma in situ. Acta Cytol 1987;31:397-411.

12 Ruba S, Schoolland M, Allpress S et al. Adenocarcinoma in situ of the uterine cervix: screening and diagnostic errors in Papanicolaou smears. Cancer 2004;102: 280-287.

13 Krane JF, Granter SR, Trask CE et al. Papanicolaou smear sensitivity for the detection of adenocarcinoma of the cervix. A study of 49 cases. Cancer (Cancer Cytopathol) 2001;93:8-15.
14 Kurman RJ, Solomon D. The Bethesda System for Reporting Cervical/Vaginal Cytologic Diagnoses. New York, NY, USA: Springer, 1994.

15 Solomon D, Nayar R. The Bethesda System for Reporting Cervical/Vaginal Cytologic Diagnoses, 2nd (edn). New York, NY, USA: Springer, 2003.

16 Ward KK, Shah NR, Saenz CC et al. Changing demographics of cervical cancer in the United States (1973-2008). Gynecol Oncol 2012;126:330-333.

17 Hildesheim A, Berrington de Gonzalez A. Etiology and prevention of cervical adenocarcinomas. J Natl Cancer Inst 2006;98:292-293.

18 McCluggage WG. New developments in endocervical glandular lesions. Histopathology 2013;62:138-160.

19 Howitt BE, Herfs M, Brister K et al. Intestinal-type endocervical adenocarcinoma in situ: an immunophenotypically distinct subset of AIS affecting older women. Am J Surg Pathol 2013;37:625-633.

20 Wilbur DC, Colgan TJ, Ferenczy AS et al. Chapter 7. Glandular tumours and precursors, part of tumours of the uterine cervix. In: Kurman RJ, Carcangiu ML, Herrington CS et al. (eds). WHO Classification of Tumours of Female Reproductive Organs, 4th (edn). Lyon, France: IARC, 2014, pp 183-194.

21 Hagiwara T, Kaku T, Kobayashi H et al. Well-differentiated villoglandular adenocarcinoma of the uterine cervix: assessment of cytological features by histological subtypes. Acta Cytol 2013;57:61-68.

22 Kusanagi Y, Kojima A, Mikami Y et al. Absence of high- risk human papillomavirus (HPV) detection in endocervical adenocarcinoma with gastric morphology and phenotype. Am J Pathol 2010;177:2169-2175.

23 Mathers ME, Johnson SJ, Wadehra V. How predictive is a cervical smear suggesting glandular neoplasia? Cytopathology 2002;13:83-91.

24 Nayar R, Wilbur DC. The Bethesda System for Reporting Cervical/Vaginal Cytologic Diagnoses, 3rd (edn). New York, NY, USA: Springer, 2015.

25 Babkowski RC, Wilbur DC, Rutkowski MA et al. The effects of endocervical canal topography, tubal metaplasia, and high canal sampling on the cytologic presentation of non-neoplastic endocervical cells. Am J Clin Pathol 1996;105:403-410.

26 Massad S, Einstein MH, Huh WK et al. 2012 Updated consensus guidelines for the management of abnormal cervical cancer screening tests and cancer precursors. J Low Genit Tract Dis 2013;17:S1-S27. 